\title{
Online Non-preemptive Scheduling on Unrelated Machines with Rejections
}

\author{
Giorgio Lucarelli \\ Univ. Grenoble Alpes, CNRS, Inria, \\ Grenoble INP, LIG \\ Grenoble, France \\ giorgio.lucarelli@imag.fr
}

\author{
Benjamin Moseley \\ Carnegie Mellon University \\ moseleyb@andrew.cmu.edu
}

\author{
Nguyen Kim Thang \\ IBISC, Univ Évry, Université \\ Paris-Saclay \\ Evry, France \\ thang@ibisc.fr
}

\author{
Abhinav Srivastav \\ Dépt. d'Informatique, ENS-Paris \\ LAMSADE, Univ. Paris-Dauphine \\ Paris, France \\ abhinavsriva@gmail.com
}

\author{
Denis Trystram \\ Univ. Grenoble Alpes, CNRS, Inria, \\ Grenoble INP, LIG \\ Grenoble, France \\ denis.trystram@imag.fr
}

\begin{abstract}
When a computer system schedules jobs there is typically a significant cost associated with preempting a job during execution. This cost can be from the expensive task of saving the memory's state and loading data into and out of memory. There is a need for nonpreemptive system schedulers to avoid the costs of preemption on desktops, servers and data centers. Despite this need, there is a gap between theory and practice. Indeed, few non-preemptive online schedulers are known to have strong foundational guarantees. This gap is likely due to strong lower bounds on any online algorithm for popular objectives. Indeed, typical worst case analysis approaches, and even resource augmented approaches such as speed augmentation, result in all algorithms having poor performance guarantees.

This paper considers online non-preemptive scheduling problems in the worst-case model where the algorithm is allowed to reject a small fraction of jobs. By rejecting only few jobs, this paper shows that the strong lower bounds can be circumvented. This model can be used to discover scheduling policies with desirable worst-case guarantees. Specifically, the paper presents algorithms for minimizing the total flow-time and minimizing the total weighted flow-time plus energy under the speed-scaling mechanism. The algorithms have a small constant competitive ratio while rejecting only a constant fraction of jobs. Beyond specific results, the paper asserts that alternative models beyond speed augmentation should be explored to aid in the discovery of good schedulers in the face of the requirement of being online and non-preemptive.
\end{abstract}

\section{CCS CONCEPTS}

- Theory of computation $\rightarrow$ Scheduling algorithms; Online algorithms;

Permission to make digital or hard copies of all or part of this work for personal or classroom use is granted without fee provided that copies are not made or distributed for profit or commercial advantage and that copies bear this notice and the full citation on the first page. Copyrights for components of this work owned by others than ACM must be honored. Abstracting with credit is permitted. To copy otherwise, or republish, to post on servers or to redistribute to lists, requires prior specific permission and/or a fee. Request permissions from permissions@acm.org.

SPAA '18, fuly 16-18, 2018, Vienna, Austria

(C) 2018 Association for Computing Machinery.

ACM ISBN 978-1-4503-5799-9/18/07...\$15.00

https://doi.org/10.1145/3210377.3210402

\section{KEYWORDS}

Online algorithms; Non-preemptive scheduling; Rejections; Primaldual; Energy

ACM Reference Format:

Giorgio Lucarelli, Benjamin Moseley, Nguyen Kim Thang, Abhinav Srivastav, and Denis Trystram. 2018. Online Non-preemptive Scheduling on Unrelated Machines with Rejections. In SPAA '18: 30th ACM Symposium on Parallelism in Algorithms and Architectures, fuly 16-18, 2018, Vienna, Austria. ACM, New York, NY, USA, 10 pages. https://doi.org/10.1145/3210377.3210402

\section{INTRODUCTION}

Designing efficient system schedulers is critical for optimizing system performance. Many environments require the scheduler to be non-preemptive, ensuring each job is scheduled on a machine without interruption. The need for non-preemption arises because preemption requires saving the state of a program and writing the state to memory or disk. For large complex tasks, the overhead cost of saving state is so large that it has to be avoided entirely.

Designing theoretically efficient online non-preemptive schedulers is challenging. Strong lower bounds have been shown, even for simple instances $[7,13]$. The difficulty lies in the pessimism of assuming the algorithm is online and must be robust to all problem instances combined with irrevocable nature of scheduling nonpreemptive jobs.

In order to overcome strong theoretical barriers when designing scheduling algorithms, Kalyanasundaram and Pruhs [12] and Phillips et al. [15] proposed using resource augmentation in terms of speed augmentation and the machine augmentation, respectively. The idea is to either give the algorithm faster processors or extra machines versus the adversary. These models provide a tool to establish a theoretical explanation for the good performance of algorithms in practice. Indeed, many practical heuristics have been shown to be competitive in the online preemptive model where the algorithm is given resource augmentation. Non-preemptive environments have resisted the discovery of strong theoretical schedulers. Designing meaningful algorithms for non-preemptive problems is an important direction in scheduling [3]. Specifically, it is known that 
a non-preemptive algorithm cannot have a small reasonable competitive ratio using only speed or machine augmentation [14] for the popular average flow time objective.

Recently, Choudhury et al. [8] extended the resource augmentation model to allow rejection (some jobs need not be completed and are rejected). By combining rejection and speed augmentation, Lucarelli et al. [14] gave competitive algorithms for non-preemptive flow-time problems. An intriguing question is the power of rejection versus resource augmentation. Is there a competitive algorithm that only uses rejection? This would establish that theoretically rejection is more powerful since there are lower bounds using resource augmentation. This paper answers this question positively.

\subsection{Models, Problems and Contribution}

Non-Preemptive Total Flow-time Minimization. In this problem, we are given a set of unrelated machines $\mathcal{M}$ and jobs arrive online. Each job $j \in \mathcal{J}$ is characterized by a release time $r_{j}$ and it takes a different processing time $p_{i j}$ if it is executed on each machine $i \in \mathcal{M}$. The characteristics of each job become known to the algorithm only after its arrival. The jobs should be scheduled non-preemptively, that is a job is considered to be successfully executed only if it is executed on a machine $i \in \mathcal{M}$ for $p_{i j}$ continuous time units. Given a schedule $\mathcal{S}$, the completion time of a job $j \in \mathcal{J}$ is denoted by $C_{j}$. Then, its flow-time is defined as $F_{j}=C_{j}-r_{j}$, that is the total amount of time during which $j$ remains in the system. Our goal is to create a non-preemptive schedule that minimizes the total flow-times of all jobs, i.e., $\sum_{j} F_{j}$.

The problem has been studied in [14] in the model of speed augmentation and rejection. Specifically, Lucarelli et al. [14] gave a $O\left(1 /\left(\epsilon_{r} \cdot \epsilon_{s}\right)\right)$-competitive algorithm that uses machines with speed $\left(1+\epsilon_{s}\right)$ and reject at most $\epsilon_{r}$-fraction of jobs for arbitrarily small $\epsilon_{r}, \epsilon_{s}>0$. A natural question is whether speed augmentation is necessary. Our main result answers positively this question.

THEOREM 1.1. For the non-preemptive total flow-time minimization problem, there exists a $2\left(\frac{1+\epsilon}{\epsilon}\right)^{2}$-competitive algorithm that removes at most $2 \epsilon$ fraction of the total number of jobs, for any $\epsilon>0$.

The design and analysis of the algorithm follow the duality approach. At the release time of any job $j$, the algorithm defines the dual variables associated to the job and assigns $j$ to some machine based on this definition. The value of the dual variables associated to $j$ are selected in order to satisfy two key properties: (i) express the marginal increase of the total weighted flow-time due to the arrival of the job - the property that has been observed [2] and has become more and more popular in dual-fitting for online scheduling; and (ii) capture the information for a future decision of the algorithm whether job $j$ will be completed or rejected. Moreover, the dual variables are defined so as to stabilize the schedule and allows us to maintain a non-preemptive schedule (even with job arrivals and rejections in the future).

The decision about rejecting a job depends on the load of the recently released jobs that are waiting in the queue of each machine. The scheduler rejects a job when this load exceeds a given threshold. The rejected job is not necessarily the one that just arrived and caused the excess in the threshold. The following lemma shows that immediate rejection policies cannot improve the competitive ratio.
Lemma 1.2. Any $\epsilon$-rejection policy which has to decide the rejection or not of each job immediately upon its arrival, has a competitive ratio of $\Omega(\sqrt{\Delta})$ for the non-preemptive total flow-time minimization problem even on a single machine environment, where $\Delta$ is the ratio of the maximum over the minimum processing time in the instance and $\epsilon>0$.

Non-Preemptive Total Flow-time Plus Energy Minimization. We next consider non-preemptive scheduling in the speed scaling model. In this model, each machine $i \in \mathcal{M}$ has a power function of the form $P\left(s_{i}(t)\right)=s_{i}(t)^{\alpha}$, where $s_{i}(t)$ is the speed of the machine $i$ at time $t$ and $\alpha>1$ is a constant parameter (usually $\alpha \in(1,3]$ ). Each job $j \in \mathcal{J}$ is now characterized by its weight $w_{j}$, its release date $r_{j}$ and, for each machine $i \in \mathcal{M}$, a machine-dependent volume of execution $p_{i j}$. A non-preemptive schedule in the speed-scaling model is a schedule in which each job is processed continuously (without being interrupted) in a machine and a job has a constant speed during its execution. Note that in the model, it is allowed to process multiple jobs in parallel on the same machine. The objective is to schedule jobs non-preemptively so that minimizing the total weighted flow-time plus the energy consumed for all jobs, i.e. $\sum_{j} w_{j} F_{j}+\sum_{i} \int_{0}^{\infty}\left(s_{i}(t)\right)^{\alpha} d t$.

Building upon the resilient ideas and techniques from flow-time minimization, we derive a competitive algorithm for the problem. Note that this algorithm does not need to process multiple jobs in parallel on the same machine, although this is permissible by the described model.

THEOREM 1.3. For the non-preemptive total weighted flow-time plus energy minimization problem, there exists an $O\left(\left(1+\frac{1}{\epsilon}\right)^{\frac{\alpha}{\alpha-1}}\right)$ competitive algorithm that rejects jobs of total weight at most an $\epsilon$-fraction of the total weight of all jobs, for any $\epsilon>0$.

Non-Preemptive Energy Minimization. Subsequently, we consider the non-preemptive energy minimization scheduling problem in the speed scaling model. The setting is similar to the previous problem but a job $j \in \mathcal{J}$ now has a release date $r_{j}$, a deadline $d_{j}$ and a processing volume $p_{i j}$ if it is assigned to machine $i \in \mathcal{M}$. Every job has to be processed non-preemptively and to be completed before its deadline. The goal is to minimize the total energy consumption $\sum_{i} \sum_{t} P_{i}\left(s_{i}(t)\right)$ where $P_{i}$ is the power function of machine $i$. (In this case we consider the discrete time setting.)

No competitive algorithm is known in the non-preemptive multiplemachine environment. Despite of some similarities to the problem of minimizing energy plus flow-time, the main difference is that in the latter, one can make a trade-off between energy and flowtime and derive a competitive algorithm whereas for the energy minimization problem, one has to deal directly with a non-linear objective. The critical issue is that no linear program (LP) with relatively small integrality gap was known. In order to derive a competitive algorithm for this problem, we make use of the primaldual approach based on configuration LP recently developed in [17]. The approach consists of introducing exponential number of variables to the natural formulation in order to reduce the integrality gap. Then, in contrast to current rounding techniques based on configuration LPs, the approach maintains greedily a competitive solution in the sense of primal-dual (without solving exponential size LPs). Interestingly, using this approach, the power functions 
are not required to be convex (a crucial property for prior analyses) and the competitive ratio is characterized by a notion of smoothness defined as follows.

Definition 1.4. A set function $f: 2^{\mathcal{N}} \rightarrow \mathbb{R}^{+}$is $(\lambda, \mu)$-smooth if for any set $A=\left\{a_{1}, \ldots, a_{n}\right\} \subseteq \mathcal{N}$ and any collection $B_{1} \subseteq B_{2} \subseteq$ $\ldots \subseteq B_{n} \subseteq B \subseteq \mathcal{N}$, the following inequality holds.

$$
\sum_{i=1}^{n}\left[f\left(B_{i} \cup a_{i}\right)-f\left(B_{i}\right)\right] \leq \lambda f(A)+\mu f(B)
$$

THEOREM 1.5. Assume that all power functions are $(\lambda, \mu)$-smooth. Then, there is a $\lambda /(1-\mu)$-competitive algorithm for the non-preemptive energy minimization scheduling problem. In particular, if $P_{i}(s)=s^{\alpha_{i}}$ for $\alpha_{i} \geq 1$ then the algorithm is $O\left(\alpha^{\alpha}\right)$-competitive where $\alpha=$ $\max _{i} \alpha_{i}$.

In the following lemma we consider the case of typical power functions of the form $P(s)=s^{\alpha}$, and we show that the above result is asymptotically optimal as a function of $\alpha$.

Lemma 1.6. Any deterministic algorithm is at least $(\alpha / 9)^{\alpha}$-competitive for the non-preemptive energy minimization problem even in a single machine environment.

\subsection{Related Work}

For the online non-preemptive scheduling problem of minimizing total weighted flow-time, any algorithm has at least $\Omega(n)$ competitive ratio, even for single machine where $n$ is the number of jobs (as mentioned in [7]). In identical machine environments, Phillips et al. [15] gave a constant competitive algorithm that uses $m \log P$ machines (recall that the adversary uses $m$ machines), where $P$ is the ratio of the largest to the smallest processing time. Moreover, an $O(\log n)$-machine $O(1)$-speed algorithm that returns the optimal schedule has been presented in [15] for the unweighted flowtime objective. Epstein and van Stee [11] proposed an $\ell$-machines $O(\min \{\sqrt[\ell]{P}, \sqrt[\ell]{n}\})$-competitive algorithm for the unweighted case on a single machine. This algorithm is optimal up to a constant factor for constant $\ell$. Recently, Lucarelli et al. [14] consider the problem in the model of speed augmentation and rejection. They showed that without rejection, no algorithm is competitive even on single machine with speed arbitrarily faster than that of adversary. Moreover, they gave a scalable $O\left(1 /\left(\epsilon_{r} \cdot \epsilon_{s}\right)\right)$-competitive algorithm that uses machines with speed $\left(1+\epsilon_{s}\right)$ and reject at most $\epsilon_{r}$ fraction of jobs for arbitrarily small $\epsilon_{r}, \epsilon_{s}>0$.

For the online non-preemptive scheduling problem of minimizing total weighted flow-time plus energy, to the best of our knowledge, no competitive algorithm is known. However, the problem in the preemptive setting has been widely studied. Bansal et al. [5] gave an $O(\alpha / \log \alpha)$-competitive algorithm for weighted flow-time plus energy in a single machine where the energy function is $s^{\alpha}$. Based on linear programming and dual-fitting, Anand et al. [2] proved an $O\left(\alpha^{2}\right)$-competitive algorithm for unrelated machines. Subsequently, Nguyen [16] and Devanur and Huang [10] presented an $O(\alpha / \log \alpha)$-competitive algorithms for unrelated machines by dual fitting and primal dual approaches, respectively.

For the online non-preemptive scheduling problem of minimizing total energy consumption, no competitive algorithm is known. Even in the preemptive scheduling in which migration of jobs between machines are not allowed, no algorithm with provable performance is given. The difficulty, as mentioned earlier, is due to the integrality gap barrier of all currently known formulations. In single machine where the issue of non-migration does not exist, Bansal et al. [6] gave a $2\left(\frac{\alpha}{\alpha-1}\right)^{\alpha} e^{\alpha}$-competitive algorithm. Moreover, Bansal et al. [4] showed that no deterministic algorithm has competitive ratio less than $e^{\alpha-1} / \alpha$. Albers et al. [1] considered the case where jobs are allowed to be executed preemptively and migration between machines is permitted. For this problem, they proposed an algorithm based on the Average RATE algorithm [18] and they showed a competitive ratio of $(1+\epsilon)\left(\alpha^{\alpha} 2^{\alpha-1}+1\right)$.

\section{MINIMIZE TOTAL FLOW-TIME}

Linear Programming Formulation. In order to formulate our problem as a linear program, for each job $j \in \mathcal{J}$, machine $i \in \mathcal{M}$ and time $t \geq r_{j}$, we introduce a binary variable $x_{i j}(t)$ which is equal to one if $j$ is processed on $i$ at time $t$, and zero otherwise. We use two lower bounds on the flow-time of each job $j \in \mathcal{J}$, assuming that it is dispatched to machine $i$ : its fractional flow-time which is defined as $\int_{r_{j}}^{\infty} \frac{t-r_{j}}{p_{i j}} x_{i j}(t) d t$ (see for example [2]), and its processing time $p_{i j}=\int_{r_{j}}^{\infty} x_{i j}(t) d t$. Then, the linear programming formulation for the problem of minimizing the total flow-time follows.

$$
\begin{array}{cc}
\min \sum_{i \in \mathcal{M}} \sum_{j \in \mathcal{J}} \int_{r_{j}}^{\infty}\left(\frac{t-r_{j}}{p_{i j}}+1\right) x_{i j}(t) d t & \\
\sum_{i \in \mathcal{M}} \int_{0}^{\infty} \frac{x_{i j}(t)}{p_{i j}} d t \geq 1 & \forall j \\
\sum_{j \in \mathcal{J}} x_{i j}(t) \leq 1 & \forall i, t \\
x_{i j}(t) \in\{0,1\} & \forall i, j, t
\end{array}
$$

Note that the objective value of the above linear program is at most twice that of the optimal non-preemptive schedule. We relax the above integer linear program by replacing the integrality constraints for each $x_{i j}(t)$ with $0 \leq x_{i j}(t) \leq 1$. The dual of the relaxed linear program is the following.

$$
\begin{aligned}
\max \sum_{j \in \mathcal{T}} \lambda_{j} & -\sum_{i \in \mathcal{M}} \int_{0}^{\infty} \beta_{i}(t) & & d t \\
\frac{\lambda_{j}}{p_{i j}}-\beta_{i}(t) & \leq \frac{t-r_{j}}{p_{i j}}+1 & & \forall i, j, t \\
\lambda_{j} & \geq 0 & & \forall j \\
\beta_{i}(t) & \geq 0 & & \forall i, t
\end{aligned}
$$

In the rejection model considered in this article, we assume that the algorithm is allowed to reject some jobs. This can be interpreted in the primal linear program by considering only the variables corresponding to the non-rejected jobs, that is the algorithm does not have to satisfy the first constraint for the rejected jobs.

The Algorithm and Definition of Dual Variables. We next define the scheduling, the rejection and the dispatching policies of our algorithm which is denoted by $\mathcal{A}$. Let $\epsilon, 0<\epsilon<1$, be an arbitrarily small constant which indicates the fraction of the total number of 
jobs that will be rejected. Each job is immediately dispatched to a machine upon its arrival. Let $U_{i}(t)$ be the set of pending jobs at time $t$ dispatched to machine $i \in \mathcal{M}$, that is the jobs dispatched to $i$ that have been released but not yet completed or rejected at time $t$. Moreover, let $q_{i j}(t)$ be the remaining processing time at time $t$ of a job $j \in \mathcal{J}$ which has been dispatched to the machine $i$.

Let $k$ be the job that is executed on machine $i$ at time $t$. We always consider the jobs in $U_{i}(t) \backslash\{k\}$ sorted in non-decreasing order with respect to their processing times; in case of ties, we consider the jobs in earliest release time order. We say that a job $j \in U_{i}(t) \backslash\{k\}$ precedes (resp. succeeds) a job $\ell \in U_{i}(t) \backslash\{k\}$ if $j$ appears before (resp. after) $\ell$ in the above order, and we write $j<\ell$ (resp. $j>\ell$ ). We use the symbols $\leq$ and $\geq$ to express the fact that $j$ may coincide with $\ell$. The scheduling policy of the algorithm $\mathcal{A}$ is the following: whenever a machine $i \in \mathcal{M}$ becomes idle at a time $t$, schedule on $i$ the job $j \in U_{i}(t)$ that precedes any other job in $U_{i}(t)$.

We use two different rules for defining our rejection policy. The first rule handles the arrival of a big group of jobs during the execution of a long job as in [14]. The second rule simulates and replaces the utility of speed-augmentation.

Rejection Rule 1. At the beginning of the execution of a job $j \in$ $\mathcal{J}$ on machine $i$, we introduce a counter $v_{j}$ which is initialized to zero. Whenever a job $\ell$ is dispatched to machine $i$ during the execution of $j$, we increase $v_{j}$ by 1 . Then, we interrupt and reject the job $j$ the first time when $v_{j} \geq \frac{1}{\epsilon}$.

Rejection Rule 2. For each machine $i \in \mathcal{M}$, we maintain a counter $c_{i}$ which is initialized to zero at $t=0$. Whenever a job $j$ is dispatched to a machine $i$, we increase $c_{i}$ by 1 . Then, we reject the job with the largest processing time in $U_{i}(t) \backslash\{k\}$ the first time when $c_{i}=1+\frac{1}{\epsilon}$, and we reset $c_{i}$ to zero.

Let $\mathcal{R}$ be the set of all rejected jobs. By slightly abusing the notation, we denote the rejection time of a job $j \in \mathcal{R}$ by $C_{j}$. Moreover, we define the flow-time of a rejected job $j \in \mathcal{R}$ to be the difference between its rejection time and its arrival time, and we denote it by $F_{j}$.

At the arrival of a new job $j \in \mathcal{J}$, let $\Delta_{i j}$ be the increase in the total flow-time if we decide to dispatch the job $j$ to the machine $i$. Fix a machine $i$ and let $k$ be the job that is executed on $i$ at $r_{j}$. Then, assuming that $j$ is dispatched to $i$ (i.e., assuming that $j \in U_{i}\left(r_{j}\right)$ ), we have that

$$
\begin{aligned}
\Delta_{i j}= & q_{i k}\left(r_{j}\right) \cdot \mathbb{1}_{\{\text {if } k \text { is not rejected (due to Rule } 1)\}}+\sum_{\ell \leq j} p_{i \ell} \\
& +\sum_{\ell>j} p_{i j} \\
& -\left(q_{i k}\left(r_{j}\right)+\sum_{\ell \neq j} q_{i k}\left(r_{j}\right)\right) \cdot \mathbb{1}_{\{\text {if } k \text { is rejected due to Rule } 1\}} \\
& -\left(q_{i k}\left(r_{j}\right)+\sum_{\ell \neq j} p_{i \ell}+p_{i j_{\max }}\right) \cdot \mathbb{1}_{\left\{\text {if } j_{\max } \text { is rejected due to Rule } 2\right\}}
\end{aligned}
$$

where the first term corresponds to the flow-time of the new job $j$, the second term corresponds to the increase of the flow-time for the jobs in $U_{i}\left(r_{j}\right)$ due to the dispatching of $j$ to machine $i$, the third term corresponds to the decrease of the flow-time for the jobs in $U_{i}\left(r_{j}\right) \cup\{k\}$ due to the rejection of $k$ (according to Rule 1), and the forth term corresponds to the decrease of the flow-time of the largest job $j_{\max }$ due to its rejection (according to Rule 2). Based on the above, we define

$$
\lambda_{i j}=\frac{1}{\epsilon} p_{i j}+\sum_{\ell \leq j} p_{i \ell}+\sum_{\ell>j} p_{i j}
$$

Then, our dispatching policy is the following: at the arrival of a new job $j$ at time $r_{j}$, dispatch $j$ to the machine $i^{*}=\operatorname{argmin}_{i \in \mathcal{M}} \lambda_{i j}$.

The quantity $\lambda_{i j}$ is strongly related with the marginal increase $\Delta_{i j}$. However, all negative terms that appear in $\Delta_{i j}$ have been eliminated in $\lambda_{i j}$. Moreover, the positive quantity $q_{i k}\left(r_{j}\right)$ does not appear in $\lambda_{i j}$, but we have added the term $\frac{1}{\epsilon} p_{i j}$. The intuition for the definition of $\lambda_{i j}$ is to charge an upper bound to the marginal increase $\Delta_{i j}$ to the $\lambda_{i \ell}$ quantities of some jobs dispatched to $i$. Specifically, the quantity $\sum_{\ell \leq j} p_{i \ell}+\sum_{\ell>j} p_{i j}$ is charged to $\lambda_{i j}$. If the positive quantity $q_{i k}\left(r_{j}\right)$ exists, then it is charged to the term $\frac{1}{\epsilon} p_{i k}$ of $\lambda_{i k}$ (i.e., to the job $k$ that is executed on $i$ at the arrival of $j)$. The rejection Rule 1 guarantees that this term is sufficient for all jobs arrived and dispatched to $i$ during the execution of $k$.

In order to deal with the ignored negative terms, we expand the notion of completion time of each job $j \in \mathcal{J}$. Let $D_{j}$ be the set of jobs that are rejected due to Rule 1 after the release time of $j$ and before its completion or rejection (including $j$ in case it is rejected), that is the jobs that cause a decrease to the flow time of $j$ due to Rule 1 . Moreover, we denote by $j_{k}$ the job released at the moment we reject a job $k \in \mathcal{R}$. Then, we say that a job $j \in \mathcal{J}$ which is dispatched to machine $i$ is definitively finished at the time

$$
\begin{aligned}
\tilde{C}_{j}= & C_{j}+\sum_{k \in D_{j}} q_{i k}\left(r_{j_{k}}\right) \\
& +\left(q_{i k}\left(r_{j_{j}}\right)+\sum_{\ell \neq j_{j}} p_{i \ell}+p_{i j}\right) \cdot \mathbb{1}_{\{\text {if } j \text { is rejected due to Rule } 2\}}
\end{aligned}
$$

Let $V_{i}(t)$ be the set of jobs that are completed or rejected at time $t$ but not yet definitively finished. Intuitively, at the completion or rejection of job $j$ at time $C_{j}$ is moved from the set of pending jobs $U_{i}(t)$ to the set of not yet definitively finished jobs $V_{i}(t)$, and it remains to this set until the time $\tilde{C}_{j}$. Let $R_{i}(t) \subseteq V_{i}(t)$ be the set of jobs that are already rejected due to Rule 2 at time $t$ but they are not yet definitively finished.

It remains to formally define the dual variables. At the arrival of a job $j \in \mathcal{J}$, we set $\lambda_{j}=\frac{\epsilon}{1+\epsilon} \min _{i \in \mathcal{M}} \lambda_{i j}$ and we never change this value again. Moreover, for each $i \in \mathcal{M}$ and $t \geq 0$, we set $\beta_{i}(t)=\frac{\epsilon}{(1+\epsilon)^{2}}\left(\left|U_{i}(t)\right|+\left|V_{i}(t)\right|\right)$. Note that, given any fixed time $t$, $\beta_{i}(t)$ may increase if a new job arrives at any time $t^{\prime}<t$. However, $\beta_{i}(t)$ never decreases in the case of rejection since the rejected jobs are transferred to the set $V_{i}(t)$ where they remain until they are definitively finished.

Analysis. We first show the following lemma which relates all but $c_{i}$ jobs in $U_{i}(t)$ to some jobs in $R_{i}(t)$.

Lemma 2.1. Fix a machine $i$ and a time $t$. Consider the jobs in $R_{i}(t)$ sorted in non-decreasing order of the time they are definitively finished; let $k_{1}, k_{2}, \ldots, k_{r}$ be this order, wherer $=\left|R_{i}(t)\right|$. There is a partition of the jobs in $U_{i}(t)$ into at most $r+1$ subsets, $U_{i}^{1}(t), U_{i}^{2}(t), \ldots, U_{i}^{r+1}(t)$ such that
(i) $\left|U_{i}^{\ell}(t)\right| \leq \frac{1}{\epsilon}$, for $1 \leq \ell \leq r$,
(ii) $\left|U_{i}^{r+1}(t)\right| \leq c_{i}$, 
(iii) for each job $j \in U_{i}^{\ell}(t), 1 \leq \ell \leq r$, the estimated completion time of $j$ assuming that no other job is released after time $t$ is at most $\tilde{C}_{k_{\ell}}$.

Proof. The proof is based on induction on time. We consider only times which correspond to discrete events that modify the sets $U_{i}(t)$ and $R_{i}(t)$, i.e., arrival of a new job, completion of a job, rejection of a job according to Rule 2 and definitive finish of a job in $R_{i}(t)$.

At the arrival of the first job dispatched to machine $i$, we have that $c_{i}=1$ and the statement directly holds. Let us assume that the partition exists at an event which occurs at time $t$. We will show that this holds also for the next event at time $t^{\prime} \geq t$. We consider the following three cases.

- If a job $j$ completes at time $t^{\prime}$, then $j$ is removed from $U_{i}\left(t^{\prime}\right)$ without affecting the mapping implied by the statement of the lemma.

- If a job $j$ arrives at time $t^{\prime}$, then $c_{i}$ is increased by one. Let $j_{\ell}$, $1 \leq \ell \leq r+1$, be the job with the largest processing time in $U_{i}^{\ell}(t)$. If $p_{j} \geq p_{j_{r}}$, then we set $U_{i}^{\ell}\left(t^{\prime}\right)=U_{i}^{\ell}(t)$ for $1 \leq \ell \leq r$ and $U_{i}^{r+1}\left(t^{\prime}\right)=U_{i}^{r+1}(t) \cup\{j\}$ and the partition is valid since $c_{i}$ is increased. Otherwise, find the biggest $z, 1 \leq z \leq \ell$, such that $p_{j}<p_{j_{z}}$. We set $U_{i}^{\ell}\left(t^{\prime}\right)=U_{i}^{\ell}(t)$ for $1 \leq \ell \leq z-1, U_{i}^{z}\left(t^{\prime}\right)=$ $U_{i}^{z}(t) \cup\{j\} \backslash\left\{j_{z}\right\}$, and $U_{i}^{\ell}\left(t^{\prime}\right)=U_{i}^{\ell}(t) \cup\left\{j_{\ell-1}\right\} \backslash\left\{j_{\ell}\right\}$ for $z+1 \leq$ $\ell \leq r+1$. By these definitions, the first two items of the lemma are satisfied by the induction hypothesis since each set, except for $U_{i}^{r+1}$, has the same size at times $t$ and $t^{\prime}$. For item (iii), we observe that the job that is added in each set $U_{i}^{\ell}, z \leq \ell \leq r$, has a shorter processing time than the job which is removed. Hence, the item (iii) holds by the definition of the scheduling policy. Moreover, if a job $k$ is rejected according to Rule 2 at time $t^{\prime}$, then $R_{i}\left(t^{\prime}\right)=R_{i}(t) \cup\{k\}$ and $U_{i}^{\left|R_{i}\left(t^{\prime}\right)\right|}\left(t^{\prime}\right)=U_{i}^{r+1}(t)$. Therefore, the lemma holds since $c_{i} \leq 1+\frac{1}{\epsilon}$ and $k$ is the job with the largest processing time (and hence the largest estimated completion time) in $U_{i}^{r+1}(t)$.

- If the job $k_{1}$ is definitively finished at time $t^{\prime}$, then assume that $U_{i}^{1}(t)$ is not empty. Then, by the induction hypothesis each job $j \in U_{i}^{1}(t)$ should complete before $t^{\prime}$, which is a contradiction to the fact that $t^{\prime}$ is the next event after $t$.

Therefore, the lemma follows.

The following corollary is an immediate consequence of Lemma 2.1.

COROLlary 2.2. For each $t$, it holds that $\left|U_{i}(t)\right| \leq \frac{1}{\epsilon}\left(\left|R_{i}(t)\right|+1\right)$.

The following lemma guarantees that the definition of the dual variables lead always to a feasible solution for the dual program.

LemmA 2.3. For all $i \in \mathcal{M}, j \in \mathcal{J}$ and $t \geq r_{j}$, the dual constraint is feasible.

Proof. For a machine $i$ and a job $j$, observe that for any fixed $t \geq r_{j}$, the value of $\beta_{i}(t)$ may only increase during the execution of the algorithm. Hence, it is sufficient to prove the constraint assuming that no job arrives after $r_{j}$. Assume that the job $k$ is executed on the machine $i$ at the arrival of the job $j$. We have the following cases.
Case 1: The job $k$ is executed at $t$. By the definition of $\lambda_{j}$ and $\lambda_{i j}$, we have:

$$
\begin{aligned}
\frac{\lambda_{j}}{p_{i j}} & \leq \frac{\epsilon}{1+\epsilon}\left(\frac{1}{\epsilon}+\frac{1}{p_{i j}} \sum_{\ell \leq j} p_{i \ell}+\sum_{\ell>j} 1\right) \leq \frac{\epsilon}{1+\epsilon}\left(\frac{1}{\epsilon}+\sum_{\ell \leq j} 1+\sum_{\ell>j} 1\right) \\
& \left(\text { since } p_{i \ell} \leq p_{i j} \text { for all } \ell \leq j\right) \\
& \leq \frac{\epsilon}{1+\epsilon}\left(\frac{1}{\epsilon}+\left|U_{i}(t)\right|+\frac{t-r_{j}}{p_{i j}}\right) \\
& \text { (since } \left.t-r_{j} \geq 0\right)
\end{aligned}
$$

Case 2: $\mathrm{A}$ job $z \leq j$ is executed at $t$. Then, we have $t-r_{j} \geq \sum_{\ell<z} p_{i \ell}$. Using the definition of $\lambda_{j}$ and $\lambda_{i j}$, we have:

$$
\begin{aligned}
\frac{\lambda_{j}}{p_{i j}} & \leq \frac{\epsilon}{1+\epsilon}\left(\frac{1}{\epsilon}+\frac{1}{p_{i j}} \sum_{\ell \leq j} p_{i \ell}+\sum_{\ell>j} 1\right) \\
& =\frac{\epsilon}{1+\epsilon}\left(\frac{1}{\epsilon}+\frac{1}{p_{i j}} \sum_{\ell<z} p_{i \ell}+\frac{1}{p_{i j}} \sum_{z \leq \ell \leq j} p_{i \ell}+\sum_{\ell>j} 1\right) \\
& \leq \frac{\epsilon}{1+\epsilon}\left(\frac{1}{\epsilon}+\frac{t-r_{j}}{p_{i j}}+\sum_{z \leq \ell \leq j} 1+\sum_{\ell>j} 1\right) \\
& \left(\text { since } p_{i \ell} \leq p_{i j} \text { for all } \ell \leq j\right) \\
& \leq \frac{\epsilon}{1+\epsilon}\left(\frac{1}{\epsilon}+\frac{t-r_{j}}{p_{i j}}+\left|U_{i}(t)\right|\right)
\end{aligned}
$$

Case 3: A job $z>j$ is executed at $t$. Then, we have $t-r_{j} \geq \sum_{\ell<z} p_{i \ell}$. Using the definition of $\lambda_{j}$ and $\lambda_{i j}$, we have:

$$
\begin{aligned}
\frac{\lambda_{j}}{p_{i j}} & \leq \frac{\epsilon}{1+\epsilon}\left(\frac{1}{\epsilon}+\frac{1}{p_{i j}} \sum_{\ell \leq j} p_{i \ell}+\sum_{\ell>j} 1\right) \\
& =\frac{\epsilon}{1+\epsilon}\left(\frac{1}{\epsilon}+\frac{1}{p_{i j}} \sum_{\ell<j} p_{i \ell}+\sum_{j<\ell<z} \frac{p_{i \ell}}{p_{i \ell}}+\sum_{\ell \geq z} 1\right) \\
& \leq \frac{\epsilon}{1+\epsilon}\left(\frac{1}{\epsilon}+\frac{1}{p_{i j}} \sum_{\ell<j} p_{i \ell}+\sum_{j<\ell<z} \frac{p_{i \ell}}{p_{i j}}+\sum_{\ell \geq z} 1\right) \\
& \left(\text { since } p_{i \ell}>p_{i j} \text { for all } \ell>j\right) \\
& \leq \frac{\epsilon}{1+\epsilon}\left(\frac{1}{\epsilon}+\frac{t-r_{j}}{p_{i j}}+\left|U_{i}(t)\right|\right)
\end{aligned}
$$

Hence, in all the three cases we have:

$$
\begin{aligned}
\frac{\lambda_{j}}{p_{i j}} & \leq \frac{\epsilon}{1+\epsilon}\left(\frac{1}{\epsilon}+\frac{t-r_{j}}{p_{i j}}+\left|U_{i}(t)\right|\right) \\
& =\frac{\epsilon}{1+\epsilon}\left(\frac{1}{\epsilon}+\frac{t-r_{j}}{p_{i j}}+\frac{\left|U_{i}(t)\right|+\epsilon\left|U_{i}(t)\right|}{1+\epsilon}\right) \\
& \leq \frac{\epsilon}{1+\epsilon}\left(\frac{1}{\epsilon}+\frac{t-r_{j}}{p_{i j}}+\frac{\left|U_{i}(t)\right|+\left|R_{i}(t)\right|+1}{1+\epsilon}\right) \\
& (\text { by Corollary 2.2) } \\
& \leq \frac{1}{1+\epsilon}+\frac{\epsilon}{(1+\epsilon)^{2}}+\frac{\epsilon}{1+\epsilon} \frac{t-r_{j}}{p_{i j}}+\beta_{i}(t)<1+\frac{t-r_{j}}{p_{i j}}+\beta_{i}(t)
\end{aligned}
$$

and the lemma follows.

Using the above results, we next prove Theorem 1.1. 
Proof of Theorem 1.1. An immediate consequence of the definition of the two rejection rules is that the jobs rejected by algorithm $\mathcal{A}$ is at most a $2 \epsilon$-fraction of the total number of jobs in $\mathcal{J}$. By Lemma 2.3, we know that the proposed definition of the dual variables leads to a feasible dual solution. For the objective value of the dual program, by the definition of $\lambda_{j}$ and $\tilde{C}_{j}$, we have that

$$
\sum_{j \in \mathcal{J}} \lambda_{j} \geq \frac{\epsilon}{1+\epsilon} \sum_{j \in \mathcal{J}}\left(\tilde{C}_{j}-r_{j}\right)
$$

Moreover, by the definition of $U_{i}(t), V_{i}(t)$ and $\tilde{C}_{j}$, we have that

$$
\sum_{i \in \mathcal{M}} \int_{0}^{\infty} \beta_{i}(t)=\frac{\epsilon}{(1+\epsilon)^{2}} \sum_{j \in \mathcal{J}}\left(\tilde{C}_{j}-r_{j}\right)
$$

Then, the dual objective is at least

$$
\left(\frac{\epsilon}{1+\epsilon}\right)^{2} \sum_{j \in \mathcal{J}}\left(\tilde{C}_{j}-r_{j}\right)
$$

Let $F_{j}^{\mathcal{A}}$ be the flow time of a job $j \in \mathcal{J}$ in the schedule constructed by algorithm $\mathcal{A}$; recall that, for a rejected job $j \in \mathcal{R}, F_{j}^{\mathcal{A}}$ corresponds to the time between its release and its rejection. By definition, we have that $\tilde{C}_{j}-r_{j} \geq F_{j}^{\mathcal{A}}$, for each $j \in \mathcal{J}$. Therefore, taking into account that the objective value of our primal linear program is at most twice the value of an optimal non-preemptive schedule, the theorem follows.

\section{MINIMIZE TOTAL WEIGHTED FLOW TIME PLUS ENERGY}

Linear Programming Formulation. Let $\delta_{i j}=\frac{w_{j}}{p_{i j}}$ be the density of a job $j \in \mathcal{J}$ on machine $i \in \mathcal{M}$. Let $s_{i j}(t)$ be a variable that represents the speed at which the job $j \in \mathcal{J}$ is executed on machine $i \in \mathcal{M}$ at time $t$. Given a constant $\gamma$ that will be defined later, we consider the following convex programming formulation for the problem of minimizing the total weighted flow time plus energy.

$$
\begin{aligned}
& \min \sum_{i \in \mathcal{M}} \sum_{j \in \mathcal{J}} \int_{r_{j}}^{\infty} s_{i j}(t) \delta_{i j}\left(t-r_{j}+p_{i j}\right) d t \\
&+\frac{\alpha}{\gamma(\alpha-1)} \sum_{i \in \mathcal{M}} \sum_{j \in \mathcal{J}} w_{j}^{\frac{\alpha-1}{\alpha}} \int_{r_{j}}^{\infty} s_{i j}(t) d t \\
&+\sum_{i \in \mathcal{M}} \int_{r_{j}}^{\infty}\left(\sum_{j \in \mathcal{J}} s_{i j}(t)\right)^{\alpha} d t \\
& \sum_{i \in \mathcal{M}} \int_{r_{j}}^{\infty} \frac{s_{i j}(t)}{p_{i j}} d t \geq 1 \\
& s_{i j}(t) \geq 0
\end{aligned}
$$$$
\forall j \in \mathcal{J}
$$$$
\forall i \in \mathcal{M}, j \in \mathcal{J}, t \geq r_{j}
$$

The first and the second [2] terms of the objective correspond to the weighted fractional flow time whereas the third term corresponds to the total energy consumed. In order to linearize the convex energy term, we use the following property which holds for any convex function $f(x): f(x) \geq f(y)+f^{\prime}(y)(x-y)$. Thus, we can relax the objective function by replacing its last term by

$$
\sum_{i \in \mathcal{M}} \int_{0}^{\infty}(1-\alpha)\left(u_{i}(t)\right)^{\alpha} d t+\sum_{i \in \mathcal{M}} \int_{0}^{\infty} \alpha\left(u_{i}(t)\right)^{\alpha-1}\left(\sum_{j \in \mathcal{J}} s_{i j}(t)\right) d t
$$

Note that the only variables in the above formulation are $s_{i j}(t)$. The quantities $u_{i}(t)$ are constants that will be defined later. In fact, $u_{i}(t)$ 's will be treated as dual variables and they will be defined during the primal-dual procedure. The dual of the above LP is the following:

$$
\begin{gathered}
\max \sum_{j \in \mathcal{J}} \lambda_{j}+\sum_{i \in \mathcal{M}} \int_{0}^{\infty}(1-\alpha)\left(u_{i}(t)\right)^{\alpha} d t \\
\frac{\lambda_{j}}{p_{i j}} \leq \delta_{i j}\left(t-r_{j}+p_{i j}\right)+\alpha\left(u_{i}(t)\right)^{\alpha-1}+\frac{\alpha}{\gamma(\alpha-1)} w_{j}^{\frac{\alpha-1}{\alpha}} \\
\forall i \in \mathcal{M}, j \in \mathcal{J}, t \geq r_{j}
\end{gathered}
$$

The Algorithm and Definition of Dual Variables. In this section, we define the scheduling, the rejection and the dispatching policies of our algorithm which is denoted by $\mathcal{A}$. Let $0<\epsilon<1$ be some arbitrarily small constant which corresponds to the fraction of the rejected weights. Each job is immediately dispatched to some machine $i \in \mathcal{M}$ upon its arrival. Let $U_{i}(t)$ be the set of pending jobs at time $t$ dispatched to machine $i \in \mathcal{M}$, that is the jobs dispatched to $i$ that have been released but not yet completed or rejected at time $t$. Moreover, let $q_{i j}(t)$ be the remaining volume at time $t$ of job $j$ which is dispatched to machine $i$.

Let $k$ be the job that is being executed on machine $i$ at time $t$. We consider the jobs in $U_{i}(t) \backslash\{k\}$ sorted in non-increasing order with respect to their densities; in case of ties, we consider the jobs in earliest release time order. We say that a job $j \in U_{i}(t) \backslash\{k\}$ precedes (resp. succeeds) a job $\ell \in U_{i}(t) \backslash\{k\}$ if $j$ appears before (resp. after) $\ell$ in the above order, and we write $j<\ell$ (resp. $j>\ell$ ). We use the symbols $\leq$ and $\geq$ to express the fact that $j$ may coincide with $\ell$.

The scheduling policy of the algorithm $\mathcal{A}$ is the following: whenever a machine $i \in \mathcal{M}$ becomes idle at a time $t$, schedule on $i$ the job $j \in U_{i}(t)$ that precedes any other job in $U_{i}(t)$. The speed of the machine $i$ at the start time $j$ is defined as $s_{i j}=\gamma\left(\sum_{\ell \in U_{i}(t)} w_{\ell}\right)^{1 / \alpha}$. Note that, the speed of $i$ is defined at the beginning of the execution of $j$ and does not change until $j$ is completed or rejected. Assuming that no other jobs arrive in the future, we can compute the expected speed of each remaining pending job $\ell \in U_{i}(t)$ which is equal to $\gamma\left(\sum_{\ell^{\prime} \geq \ell} w_{\ell^{\prime}}\right)^{1 / \alpha}$.

As soon as the machine $i$ starts executing a job $j$, we introduce a counter $v_{j}$ which is initialized to zero. Each time a job $\ell$ is released during the execution of $j$ and it is dispatched to machine $i$, we increase $v_{j}$ by $w_{\ell}$. Then, the rejection policy of the algorithm $\mathcal{A}$ is the following: interrupt the execution of $j$ and reject it the first time when $v_{j}>w_{j} / \epsilon$.

Assume that at the arrival of a new job $j$ at time $r_{j}$, the machine $i$ is executing the job $k$. For each $\ell \in U_{i}(t) \backslash\{k\}$, let $W_{\ell}=$ $\sum_{\ell^{\prime} \in U_{i}(t) \backslash\{k\}: \ell^{\prime} \geq \ell} w_{\ell^{\prime}}$. We denote by $\Delta_{i j}$ the marginal increase in the total weighted flow time that will occur following the scheduling and rejection policies of $\mathcal{A}$, if we decide to dispatch the job $j$ to machine $i$. Then, $\Delta_{i j}$ can be bounded as follows (we ignore the increase of the speed and hence the decrease of the processing time 
for each job $\ell<j$ )

$$
\Delta_{i j} \leq\left\{\begin{array}{l}
w_{j}\left(\frac{q_{i k}\left(r_{j}\right)}{s_{k}}+\sum_{\ell \leq j} \frac{p_{i \ell}}{\gamma W_{\ell}^{1 / \alpha}}\right)+\left(\sum_{\ell>j} w_{\ell}\right) \frac{p_{i j}}{\gamma W_{j}^{1 / \alpha}} \\
\quad \text { if } v_{k}+w_{j} \leq \frac{w_{k}}{\epsilon} \\
w_{j}\left(\sum_{\ell \leq j} \frac{p_{i \ell}}{\gamma W_{\ell}^{1 / \alpha}}\right)+\left(\sum_{\ell>j} w_{\ell}\right) \frac{p_{i j}}{\gamma W_{j}^{1 / \alpha}}-\left(\sum_{\ell \neq j} w_{\ell}\right) \frac{q_{i k}\left(r_{j}\right)}{s_{k}}
\end{array}\right.
$$

where in both cases, the first positive term correspond to the weighted flow time of the job $j$, while the second positive term correspond to the marginal increase of the weighted flow time of other jobs, that is the completion time of the jobs with density smaller than the density of $j$ is delayed by $p_{i j} / \gamma W_{j}^{1 / \alpha}$. The negative term in the second case corresponds to the decrease in the weighted flow time of all jobs in $U_{i}(t)$ if the job $k$ is rejected. Then, we define a set of variables $\lambda_{i j}$, for all $i \in \mathcal{M}$, as: $\lambda_{i j}=$ $w_{j}\left(\frac{p_{i j}}{\epsilon}+\sum_{\ell \leq j} \frac{p_{i \ell}}{\gamma W_{\ell}^{1 / \alpha}}\right)+\left(\sum_{\ell>j} w_{\ell}\right) \frac{p_{i j}}{\gamma W_{j}^{1 / \alpha}}$. The dispatching pol$i c y$ is the following: dispatch the job $j$ to the machine $i^{*}$ such that $i^{*}=\operatorname{argmin}_{i \in \mathcal{M}}\left\{\lambda_{i j}\right\}$.

We next define the dual variables $\lambda_{j}$ as well as the quantities $u_{i}(t)$. Based on the dispatching policy, we set $\lambda_{j}=\frac{\epsilon}{1+\epsilon} \min _{i \in \mathcal{M}}\left\{\lambda_{i j}\right\}$. For each job $j$, let $D_{j}$ be the set of the jobs rejected due to the rejection policy between $r_{j}$ and the time when $j$ is completed or rejected. Let $j_{k}$ denote the job released at the time when our policy rejects the job $k$. Then, we say that a job $j$ is definitively finished at the time $\sum_{k \in D_{j}} \frac{q_{i k}\left(r_{j_{k}}\right)}{s_{k}}$ after its completion or rejection. For every job $\ell$, define the fractional weight $w_{\ell}(t)$ of $\ell$ at time $t$ as $w_{\ell} q_{i \ell}(t) / p_{i \ell}$. Let $Q_{i}(t)$ be the set of jobs that are dispatched to machine $i$ and are already completed or rejected but no yet definitively finished at time $t$. Let $V_{i}(t)=\sum_{\ell \in U_{i}(t) \cup Q_{i}(t)} w_{\ell}(t)$ be the total fractional weight of jobs that are not definitively finished on machine $i$ at time $t$. We define $u_{i}(t)$ as follows: $u_{i}(t)=\left(\frac{\epsilon}{\gamma(1+\epsilon)(\alpha-1)}\right)^{\frac{1}{\alpha-1}} V_{i}(t)^{1 / \alpha}$. Note that when a job is rejected, it is transferred from $U_{i}(t)$ to $Q_{i}(t)$ where it remains until the time it is definitively finished.

Consider now two sets of jobs $I_{1}$ and $I_{2}$ assigned to machine $i$ such that they are identical except that there is only a job $j \in I_{1} \backslash I_{2}$. Moreover, assume that no job is released after time $r_{j}$ in either of the instances. Then the algorithm $\mathcal{A}$ is said to be monotonic iff $\sum_{\ell \in I_{2}} w_{\ell}(t) \leq \sum_{\ell \in I_{1}} w_{\ell}(t), \forall t$ where the jobs in $I_{1}$ and $I_{2}$ are scheduled according to $\mathcal{A}$. The following lemma shows the monotonicity of $V_{i}(t)$.

\section{LEMMA 3.1. $V_{i}(t)$ is monotone for every machine $i$.}

Proof. Let $k$ be the job executing on machine $i$ at time $t$. Observe that $V_{i}(t)$ changes due to the arrival of a new job. Assume that a new job $j$ arrives at $t=r_{j}$. Then, it is sufficient to show that $V_{i}(t)$ is non-decreasing during anytime $t^{\prime} \geq t$. Consider the jobs in $U_{i}(t) \backslash\{k\}$. Since all such jobs are scheduled in non-increasing order of their densities, the total fractional weight of jobs in $U_{i}(t) \backslash\{k\}$ is monotonic with respect to the arrival of a new job (refer to Lemma 6.1 in [2]).
In the case where $k$ is not rejected then for $t^{\prime}<t+\frac{q_{i k}\left(r_{j}\right)}{s_{j}}$, the speed of the machine $i$ is a constant. Hence, $U_{i}\left(t^{\prime}\right)$ is a constant. Using Lemma 6.1 in [2], the lemma holds for this case. In the case where $k$ is rejected then $U_{i}(t)$ decreases due to the removal of $k$. Since all jobs in $U_{i}(t) \backslash\{j\}$ remain for at least $\frac{q_{i k}\left(r_{j}\right)}{s_{j}}$ time in $Q_{i}(t)$ after their completion or rejection from $U_{i}(t)$, the total fractional weight of jobs in $U_{i}(t) \cup Q_{i}(t)$ is monotonic with respect to the rejection of job $k$. Using this property with Lemma 6.1 in [2], the lemma holds.

Analysis. The following lemma guarantees that the definition of the dual variables lead always to a feasible solution for the dual program.

Lemma 3.2. For every $i \in \mathcal{M}, j \in \mathcal{J}$ and $t \geq r_{j}$, the dual constraint is feasible.

Proof. Fix a machine $i$. By Lemma 3.1, $u_{i}(t)$ 's do never decrease during the execution of the algorithm. Hence, it is sufficient to prove the inequality for the job $j$ at time $r_{j}$. Let $k$ be the job executed in machine $i$ at $r_{j}$. Moreover, let $\bar{C}_{j}$ be the completion time of the job $j$ estimated at time $r_{j}$ if it is assigned to machine $i$. Specifically, if $k$ is rejected then $\bar{C}_{j}=r_{j}+\sum \ell \leq j \frac{p_{i \ell}}{\gamma W_{\ell}^{1 / \alpha}}$; otherwise we have $\bar{C}_{j}=r_{j}+\frac{q_{i k}\left(r_{j}\right)}{s_{k}}+\sum_{\ell \leq j} \frac{p_{i \ell}}{\gamma W_{\ell}^{1 / \alpha}}$.

By the definitions of $\lambda_{j}$ and $\lambda_{i j}$, we have:

$\frac{\lambda_{j}}{p_{i j}} \leq \frac{\epsilon}{1+\epsilon} \frac{\lambda_{i j}}{p_{i j}}=\frac{\epsilon}{1+\epsilon}\left(\frac{w_{j}}{p_{i j}}\left(\frac{p_{i j}}{\epsilon}+\sum_{\ell \leq j} \frac{p_{i \ell}}{\gamma W_{\ell}^{1 / \alpha}}\right)+\left(\sum_{\ell>j} w_{\ell}\right) \frac{1}{\gamma W_{j}^{1 / \alpha}}\right)$

Let $w_{n}$ denote the weight of the latest job according to the precedence order defined above.

Case 1: $t \leq \bar{C}_{j}$. Assume, first, that the job $k$ is running at time $t$. Hence, we have that

$$
t-r_{j}=\frac{q_{i k}\left(r_{j}\right)-q_{i k}(t)}{s_{k}}
$$

and thus

$$
\begin{aligned}
\frac{\lambda_{j}}{p_{i j}}- & \delta_{i j}\left(t-r_{j}+p_{i j}\right) \\
\leq & \frac{\epsilon}{1+\epsilon}\left(\frac{w_{j}}{p_{i j}}\left(\frac{p_{i j}}{\epsilon}+\sum_{\ell \leq j} \frac{p_{i \ell}}{\gamma W_{\ell}^{1 / \alpha}}\right)+\left(\sum_{\ell>j} w_{\ell}\right) \frac{1}{\gamma W_{j}^{1 / \alpha}}\right) \\
& -\frac{w_{j}}{p_{i j}}\left(\frac{q_{i k}\left(r_{j}\right)-q_{i k}(t)}{s_{k}}+p_{i j}\right) \\
\leq & \frac{\epsilon}{1+\epsilon}\left(\frac{w_{j}}{p_{i j}} \sum_{\ell \leq j} \frac{p_{i \ell}}{\gamma W_{\ell}^{1 / \alpha}}+\left(\sum_{\ell>j} w_{\ell}\right) \frac{1}{\gamma W_{j}^{1 / \alpha}}\right) \\
& -\frac{w_{j}}{p_{i j}} \cdot \frac{q_{i k}\left(r_{j}\right)-q_{i k}(t)}{s_{k}} \\
\leq & \frac{\epsilon}{1+\epsilon}\left(\frac{w_{j}}{p_{i j}} \sum_{\ell \leq j} \frac{p_{i \ell}}{\gamma W_{\ell}^{1 / \alpha}}+\left(\sum_{\ell>j} w_{\ell}\right) \frac{1}{\gamma W_{j}^{1 / \alpha}}\right)
\end{aligned}
$$

(since $t \geq r_{j}$ and hence $q_{i k}\left(r_{j}\right)-q_{i k}(t) \geq 0$ ) 


$$
\begin{aligned}
& \leq \frac{\epsilon}{1+\epsilon}\left(\sum_{\ell \leq j} \frac{w_{\ell}}{\gamma W_{\ell}^{1 / \alpha}}+\left(\sum_{\ell>j} w_{\ell}\right) \frac{1}{\gamma W_{j}^{1 / \alpha}}\right) \\
& \text { since } \left.\frac{w_{\ell}}{p_{i \ell}} \geq \frac{w_{j}}{p_{i j}} \text { for any } \ell \leq j\right) \\
& \leq \frac{\epsilon}{1+\epsilon}\left(\sum_{\ell \leq j} \frac{w_{\ell}}{\gamma W_{\ell}^{1 / \alpha}}+\sum_{\ell>j} \frac{w_{\ell}}{\gamma W_{\ell}^{1 / \alpha}}\right) \\
& =\frac{\epsilon}{1+\epsilon} \sum_{\ell \neq k} \frac{w_{\ell}}{\gamma W_{\ell}^{1 / \alpha}} \\
& \leq \frac{\epsilon}{1+\epsilon} \int_{w_{n}}^{V_{i}(t)+w_{j}} \frac{d z}{\gamma z^{1 / \alpha}} \\
& =\frac{\epsilon}{1+\epsilon} \cdot \frac{\alpha}{\gamma(\alpha-1)}\left(V_{i}(t)+w_{j}\right)^{\frac{\alpha-1}{\alpha}} \\
& \leq \frac{\epsilon}{1+\epsilon} \cdot \frac{\alpha}{\gamma(\alpha-1)}\left(V_{i}(t)^{\frac{\alpha-1}{\alpha}}+w_{j}^{\frac{\alpha-1}{\alpha}}\right) \\
& =\frac{\epsilon}{1+\epsilon} \cdot \frac{\alpha}{\gamma(\alpha-1)}\left(\frac{\gamma(1+\epsilon)(\alpha-1)}{\epsilon}\left(u_{i}(t)\right)^{\alpha-1}+w_{j}^{\frac{\alpha-1}{\alpha}}\right) \\
& =\alpha\left(u_{i}(t)\right)^{\alpha-1}+\frac{\epsilon}{1+\epsilon} \cdot \frac{\alpha}{\gamma(\alpha-1)} w_{j}^{\frac{\alpha-1}{\alpha}} \\
& \leq \alpha\left(u_{i}(t)\right)^{\alpha-1}+\frac{\alpha}{\gamma(\alpha-1)} w_{j}^{\frac{\alpha-1}{\alpha}}
\end{aligned}
$$

Assume now that a job $h \neq k$ is executing at time $t$. Therefore, the machine $i$ has processed all the jobs which have density higher than $\delta_{i h}$. Moreover, the job $k$ is either completed or rejected. Hence, we have that

$$
t-r_{j} \geq \sum_{\ell<h} \frac{p_{i \ell}}{\gamma W_{\ell}^{1 / \alpha}}+\frac{p_{i h}-q_{i h(t)}}{\gamma W_{h}^{1 / \alpha}}
$$

and thus

$$
\begin{aligned}
& \frac{\lambda_{j}}{p_{i j}}-\delta_{i j}\left(t-r_{j}+p_{i j}\right) \\
& \leq \frac{\epsilon}{1+\epsilon}\left(\frac{w_{j}}{p_{i j}}\left(\frac{p_{i j}}{\epsilon}+\sum_{\ell \leq j} \frac{p_{i \ell}}{\gamma W_{\ell}^{1 / \alpha}}\right)+\left(\sum_{\ell>j} w_{\ell}\right) \frac{1}{\gamma W_{j}^{1 / \alpha}}\right) \\
& -\frac{w_{j}}{p_{i j}}\left(\sum_{\ell<h} \frac{p_{i \ell}}{\gamma W_{\ell}^{1 / \alpha}}+\frac{p_{i h}-q_{i h(t)}}{\gamma W_{h}^{1 / \alpha}}+p_{i j}\right) \\
& =\frac{\epsilon}{1+\epsilon}\left(\frac{w_{j}}{p_{i j}} \sum_{h \leq \ell \leq j} \frac{p_{i \ell}}{\gamma W_{\ell}^{1 / \alpha}}+\left(\sum_{\ell>j} w_{\ell}\right) \frac{1}{\gamma W_{j}^{1 / \alpha}}\right) \\
& -\frac{w_{j}}{p_{i j}}\left(\frac{1}{1+\epsilon} \cdot \sum_{\ell<h} \frac{p_{i \ell}}{\gamma W_{\ell}^{1 / \alpha}}-\frac{\epsilon}{1+\epsilon} p_{i j}-\frac{p_{i h}-q_{i h(t)}}{\gamma W_{h}^{1 / \alpha}}\right) \\
& \leq \frac{\epsilon}{1+\epsilon}\left(\sum_{h \leq \ell \leq j} \frac{w_{\ell}}{\gamma W_{\ell}^{1 / \alpha}}+\sum_{\ell>j} \frac{w_{\ell}}{\gamma W_{\ell}^{1 / \alpha}}\right) \\
& =\frac{\epsilon}{1+\epsilon} \sum_{\ell \geq h} \frac{w_{\ell}}{\gamma W_{\ell}^{1 / \alpha}} \leq \frac{\epsilon}{1+\epsilon} \int_{w_{n}}^{V_{i}(t)+w_{j}} \frac{d_{z}}{\gamma z^{1 / \alpha}} \\
& \leq \alpha\left(u_{i}(t)\right)^{\alpha-1}+\frac{\alpha}{\gamma(\alpha-1)} w_{j}^{\frac{\alpha-1}{\alpha}}
\end{aligned}
$$

Case 2: $t>\bar{C}_{j}$. Let $h$ be the job executing at time $t$. Thus, the machine $i$ has processed all the jobs which have density higher than $\delta_{i h}$. Hence, we have

$$
t-r_{j} \geq \sum_{\ell<h} \frac{p_{i \ell}}{\gamma W_{\ell}^{1 / \alpha}}+\frac{p_{i h}-q_{i h}(t)}{\gamma W_{h}^{1 / \alpha}}
$$

Thus

$$
\begin{aligned}
\frac{\lambda_{j}}{p_{i j}}- & \delta_{i j}\left(t-r_{j}+p_{i j}\right) \\
\leq & \frac{\epsilon}{1+\epsilon}\left(\frac{w_{j}}{p_{i j}}\left(\frac{p_{i j}}{\epsilon}+\sum_{\ell \leq j} \frac{p_{i \ell}}{\gamma W_{\ell}^{1 / \alpha}}\right)+\left(\sum_{\ell>j} w_{\ell}\right) \frac{1}{\gamma W_{j}^{1 / \alpha}}\right) \\
& -\frac{w_{j}}{p_{i j}}\left(\sum_{\ell<h} \frac{p_{i \ell}}{\gamma W_{\ell}^{1 / \alpha}}+\frac{p_{i h}-q_{i h(t)}}{\gamma W_{h}^{1 / \alpha}}+p_{i j}\right) \\
= & \frac{\epsilon}{1+\epsilon}\left(\frac{w_{j}}{p_{i j}} \sum_{\ell \leq j} \frac{p_{i \ell}}{\gamma W_{\ell}^{1 / \alpha}}+\left(\sum_{\ell>j} w_{\ell}\right) \frac{1}{\gamma W_{j}^{1 / \alpha}}\right) \\
& -\frac{w_{j}}{p_{i j}}\left(\frac{1}{1+\epsilon} \cdot \sum_{\ell<h} \frac{p_{i \ell}}{\gamma W_{\ell}^{1 / \alpha}}-\frac{\epsilon}{1+\epsilon} p_{i j}-\frac{p_{i h}-q_{i h(t)}}{\gamma W_{h}^{1 / \alpha}}\right) \\
\leq & \frac{\epsilon}{1+\epsilon}\left(\sum_{\ell>h} \frac{w_{\ell}}{\gamma W_{\ell}^{1 / \alpha}}\right) \\
= & \frac{\epsilon}{1+\epsilon} \sum_{\ell \geq h} \frac{w_{\ell}}{\gamma W_{\ell}^{1 / \alpha}} \leq \frac{\epsilon}{1+\epsilon} \int_{w_{n}}^{V_{i}(t)+w_{j}} \frac{d_{z}}{\gamma z^{1 / \alpha}} \\
\leq & \alpha\left(u_{i}(t)\right)^{\alpha-1}+\frac{\alpha-1}{\gamma(\alpha-1)} w_{j}^{\alpha}
\end{aligned}
$$

Based on this lemma we can prove Theorem 1.3.

Proof of Theorem 1.3. By Lemma 3.2, the proposed dual variables constitute a feasible solution for the dual program. Since each job $j \in \mathcal{J}$ is charged to at most one other job while a job $k$ is rejected the first time where $v_{k}>\frac{w_{k}}{\epsilon}$, the algorithm $\mathcal{A}$ rejects jobs of total weight at most $\epsilon \sum_{j \in \mathcal{J}} w_{j}$. Hence, it remains to give a lower bound for the dual objective based on the proposed dual variables.

Let $\mathcal{R}$ be the set of rejected jobs. We denote by $F_{j}^{\mathcal{A}}$ the flowtime of a job $j \in \mathcal{J} \backslash \mathcal{R}$ in the schedule of $\mathcal{A}$. By slightly abusing the notation, for a job $k \in \mathcal{R}$, we will also use $F_{k}^{\mathcal{A}}$ to denote the total time passed after $r_{k}$ until deciding to reject a job $k$, that is, if $k$ is rejected at the release of the job $j \in \mathcal{J}$ then $F_{k}^{\mathcal{A}}=r_{j}-r_{k}$. Denote by $j_{k}$ the job released at the moment we decided to reject $k$, i.e., for the counter $v_{k}$ before the arrival of job $j_{k}$ we have that $w_{k} / \epsilon-w_{j_{k}}<v_{k}<w_{k} / \epsilon$.

Let $\Delta_{j}$ be the total increase in the flow-time caused by the arrival of the job $j \in \mathcal{J}$, i.e., $\Delta_{j}=\Delta_{i j}$, where $i \in \mathcal{M}$ is the machine to which $j$ is dispatched by $\mathcal{A}$. For the objective function of the dual 
program we have

$$
\begin{aligned}
\sum_{j \in \mathcal{J}} \lambda_{j} & +\sum_{i \in \mathcal{M}} \int_{0}^{\infty}(1-\alpha)\left(u_{i}(t)\right)^{\alpha} d t \\
\geq & \frac{\epsilon}{1+\epsilon}\left(\sum_{j \in \mathcal{J}} \Delta_{j}+\sum_{k \in \mathcal{R}}\left(\frac{q_{i k}\left(r_{j_{k}}\right)}{s_{k}} \sum_{\ell \neq j_{k}} w_{\ell}\right)\right) \\
& -(\alpha-1)\left(\frac{\epsilon}{\gamma(1+\epsilon)(\alpha-1)}\right)^{\frac{\alpha}{\alpha-1}} V_{i}(t) \\
\geq & \left(\frac{\epsilon}{1+\epsilon}-(\alpha-1)\left(\frac{\epsilon}{\gamma(1+\epsilon)(\alpha-1)}\right)^{\frac{\alpha}{\alpha-1}}\right) F^{*}
\end{aligned}
$$

The total weighted flow time plus energy is

$2 F^{*}+\left(\frac{\alpha}{\gamma(\alpha-1)}\right) F^{*}+\sum_{i} \int_{0}^{\infty}\left(s_{i}(t)\right)^{\alpha} d t \leq\left(2+\left(\frac{\alpha}{\gamma(\alpha-1)}\right)+\gamma^{\alpha}\right) F^{*}$

Hence the competitive ratio is:

$$
\frac{\left(2+\left(\frac{\alpha}{\gamma(\alpha-1)}\right)+\gamma^{\alpha}\right)}{\left(\frac{\epsilon}{1+\epsilon}\right)-\left(\frac{\epsilon}{\gamma(1+\epsilon)}\right)^{\frac{\alpha}{\alpha-1}}(\alpha-1)^{\frac{-1}{\alpha-1}}}
$$

We choose $\gamma=\left(\frac{\epsilon}{1+\epsilon}\right)^{\frac{1}{\alpha-1}} \frac{1}{\alpha-1}(\alpha-1+\ln (\alpha-1))^{\frac{\alpha-1}{\alpha}}$. Observe that denominator becomes $\frac{\epsilon}{1+\epsilon}\left(\frac{\ln (\alpha-1)}{\alpha-1+\ln (\alpha-1)}\right)$ and the numerator becomes $2+2\left(\frac{1+\epsilon}{\epsilon}\right)^{\frac{1}{\alpha-1}}+\left(\frac{\epsilon}{1+\epsilon}\right)^{2}$. Hence the competitive ratio is at most $\mathrm{O}\left(\left(1+\frac{1}{\epsilon}\right)^{\frac{\alpha}{\alpha-1}}\right)$.

\section{MINIMIZE TOTAL ENERGY CONSUMPTION}

Formulation. In the problem, we consider the sets of discretized speeds $\mathcal{V}$ and times. We can do that and loose only a factor $(1+\epsilon)$ for $\epsilon$ arbitrarily small. In the non-preemptive model, the execution of a job is specified by three parameters: (1) a machine in which it is executed; (2) a starting time; and (3) a speed which is constant during its execution. Note that the parameters imply the completion time of job. A valid execution of a job $j$ must have the starting time and completion time in $\left[r_{j}, d_{j}\right]$. We say that a strategy of a job is a specification of a valid execution of the job. Formally, a strategy $s_{i, j, k}$ of a job $j$ in machine $i$ indicates the starting time of the job and its speed during the execution. Let $\mathcal{S}_{j}$ be a set of strategies of job $j$. As the sets of speeds and times are finite, so is the set of strategies $\mathcal{S}_{j}$ for every job $j$. Let $x_{i, j, k}$ be a variable indicating whether job $j$ is executed by strategy $s_{i, j, k} \in \mathcal{S}_{j}$. We say that $A$ is a configuration in machine $i$ if $A$ is a feasible schedule of a subset of jobs. Specifically, $A$ consists of tuples $(i, j, k)$ meaning that job $j$ is executed in machine $i$ following the strategy $s_{i, j, k}$. For configuration $A$ and machine $i$, let $z_{i, A}$ be a variable such that $z_{i, A}=1$ if and only if for every triple $(i, j, k) \in A, x_{i, j, k}=1$. In other words, $z_{i, A}=1$ iff the schedule in machine $i$ is exactly $A$. The energy cost of a configuration $A$ of machine $i$ is $f_{i}(A)=\sum_{t} P_{i}(A(t))$ where $A(t)$ is the speed of the corresponding schedule at time $t$. We consider the following formulation and the dual of its relaxation.

$$
\begin{array}{rlrl}
\min \sum_{i, A} f_{i}(A) z_{i, A} & \\
\sum_{i, k: s_{i, j, k} \in \mathcal{S}_{j}} x_{i, j, k} & =1 & \forall j \\
\sum_{A:(i, j, k) \in A} z_{i, A} & =x_{i, j, k} & \forall i, j, k \\
\sum_{A} z_{i, A} & =1 & \forall i \\
x_{i, j, k}, z_{i, A} & \in\{0,1\} & \forall i, j, k, A
\end{array}
$$

$$
\begin{array}{rlrl}
\max \sum_{j} \delta_{j} & +\sum_{i} \gamma_{i} & & \\
\delta_{j} & \leq \beta_{i, j, k} & \forall i, j, k \\
\gamma_{i}+\sum_{(i, j, k) \in A} \beta_{i, j, k} & \leq f_{i}(A) & \forall i, A
\end{array}
$$

In the primal, the first constraint guarantees that a job $j$ has to be processed by some valid execution (in some machine). The second constraint ensures that if job $j$ follows strategy $s_{i, j, k}$ then in the solution, the schedule (configuration) on machine $i$ must contain the execution corresponding to strategy $s_{i, j, k}$. The third constraint says that in the solution, there is always a configuration (schedule) associated to machine $i$.

Algorithm. We first interpret intuitively the dual variables, dual constraints and derive useful observations for a competitive algorithm. Variable $\delta_{j}$ represents the increase of energy to the arrival of job $j$. Variable $\beta_{i, j, k}$ stands for the marginal energy if job $j$ follows strategy $s_{i, j, k}$. By this interpretation, the first dual constraint clearly indicates the greedy behavior of an algorithm. That is, if a new job $j$ is released, select a strategy $s_{i, j, k} \in \mathcal{S}_{j}$ that minimizes the marginal increase of the total energy.

Let $A_{i}^{*}$ be the set of current schedule of machine $i$. Initially, $A_{i}^{*} \leftarrow$ $\emptyset$ for every $i$. At the arrival of job $j$, select a strategy $s_{i, j, k} \in \mathcal{S}_{j}$ that minimizes $\left[f_{i}\left(A_{i}^{*} \cup s_{i, j, k}\right)-f_{i}\left(A_{i}^{*}\right)\right]$ where $\left(A_{i}^{*} \cup s_{i, j, k}\right)$ is the current schedule plus the execution of job $j$ which follows strategy $s_{i, j, k}$. Let $s_{i^{*}, j, k^{*}}$ be an optimal strategy. Then assign job $j$ to machine $i^{*}$ and process it according to the corresponding execution of $s_{i^{*}, j, k^{*}}$. In the algorithm, we never interrupt or modify the speed of a job.

We implement this algorithm as follows. Let $u_{i t}$ be the speed of machine $i$ at time $t$. Initially, set $u_{i t} \leftarrow 0$ for every machine $i$ and time $t$. At the arrival of a job $j$, compute the minimum energy increase if $j$ is assigned to machine $i$ and is executed with constant speed. This corresponds to the optimization problem

$$
\begin{gathered}
\min _{i} \min _{\tau, v} \sum_{t=\tau}^{\tau+p_{i j} / v}\left[f_{i}\left(u_{i t}+v\right)-f_{i}\left(u_{i t}\right)\right] \\
\text { s.t } \quad r_{j} \leq \tau \leq \tau+\frac{p_{i j}}{v} \leq d_{j}, \quad v \in \mathcal{V}
\end{gathered}
$$

Dual variables. Assume that all energy power functions $f_{i}$ are $(\lambda, \mu)$-smooth for some fixed parameters $\lambda>0$ and $\mu<1$. We 
construct a dual feasible solution. Define $\delta_{j}$ as $1 / \lambda$ times the the increase of the total cost due to the arrival of job $j$. For each machine $i$ and job $j$, define $\beta_{i, j, k}:=\frac{1}{\lambda}\left[f_{i}\left(A_{i,<j}^{*} \cup s_{i, j, k}\right)-f_{i}\left(A_{i,<j}^{*}\right)\right]$ where $A_{i,<j}^{*}$ is the schedule of $i$ (due to the algorithm) prior to the arrival of $j$. Finally, for every machine $i$ define dual variable $\gamma_{i}:=-\frac{\mu}{\lambda} f_{i}\left(A_{i}^{*}\right)$ where $A_{i}^{*}$ is the schedule of machine $i$ (at the end of the instance).

\section{Lemma 4.1. The defined variables form a dual feasible solution.}

Proof. The first dual constraint follows immediately the definitions of $\delta_{j}, \beta_{i, j, k}$ and the decision of the algorithm. Specifically, the right-hand side of the constraint represents $1 / \lambda$ times the increase of energy if a job $j$ follows a strategy $s_{i, j, k}$. This is larger than $1 / \lambda$ times the minimum increase of energy optimized over all strategies in $\mathcal{S}_{j}$, which is $\delta_{j}$.

For the second constraint, fix a machine $i$ and an arbitrary configuration $A$ on machine $i$. Then,

$$
\begin{gathered}
-\frac{\mu}{\lambda} f_{i}\left(A_{i}^{*}\right)+\frac{1}{\lambda} \sum_{(i, j, k) \in A}\left[f_{i}\left(A_{i,<j}^{*} \cup s_{i, j, k}\right)-f_{i}\left(A_{i,<j}^{*}\right)\right] \leq f_{i}(A) \Leftrightarrow \\
\sum_{(i, j, k) \in A}\left[f_{i}\left(A_{i,<j}^{*} \cup s_{i, j, k}\right)-f_{i}\left(A_{i,<j}^{*}\right)\right] \leq \lambda f_{i}(A)+\mu f_{i}\left(A_{i}^{*}\right)
\end{gathered}
$$

We argue that this inequality follows the $(\lambda, \mu)$-smoothness of energy power functions. We slightly abuse notation by defining $A_{i,<j}^{*}(t)$ as the speed of machine $i$ (due to the algorithm) at time $t$ before the arrival of job $j$ and $s_{i, j, k}(t)$ be the speed at time $t$ of job $j$ if it follows the strategy $s_{i, j, k}$. Observe that $A_{i,<j}^{*}(t)$ is the sum of speeds (according to the algorithm) at time $t$ of jobs assigned to machine $i$ prior to job $j$. For any time $t$, as the power $P_{i}$ is $(\lambda, \mu)$-smooth, we have

$$
\begin{array}{r}
\sum_{(i, j, k) \in A}\left[P_{i}\left(A_{i,<j}^{*}(t)+s_{i, j, k}(t)\right)-P_{i}\left(A_{i,<j}^{*}(t)\right)\right] \\
\leq \lambda P_{i}\left(\sum_{(i, j, k) \in A} s_{i, j, k}(t)\right)+\mu P_{i}\left(A_{i}^{*}(t)\right)
\end{array}
$$

Summing over all $t$, Inequality (1) holds and the lemma follows.

We are now ready to prove the Theorem 1.5.

Proof of Theorem 1.5. By the definition of the dual variables, the dual objective is

$$
\sum_{j} \delta_{j}+\sum_{i} \gamma_{i}=\sum_{i} \frac{1}{\lambda} f_{i}\left(A_{i}^{*}\right)-\sum_{i} \frac{\mu}{\lambda} f_{i}\left(A_{i}^{*}\right)=\frac{1-\mu}{\lambda} \sum_{i} f_{i}\left(A_{i}^{*}\right)
$$

Besides, the cost of the solution due to the algorithm is $\sum_{i} f_{i}\left(A_{i}^{*}\right)$. Hence, the competitive ratio is at most $\lambda /(1-\mu)$.

In particular, the power functions of the form $P_{i}(s)=s^{\alpha_{i}}, \alpha_{i}>$ 1 , are $O\left(\alpha^{\alpha-1}, \frac{\alpha-1}{\alpha}\right)$-smooth where $\alpha=\max _{i} \alpha_{i}$. By the smooth inequalities in [9], for any sequences of non-negative real numbers $\left\{a_{1}, a_{2}, \ldots, a_{n}\right\}$ and $\left\{b_{1}, b_{2}, \ldots, b_{n}\right\}$ and for any $\alpha \geq 1$, it holds that

$$
\sum_{i=1}^{n}\left[\left(b_{i}+\sum_{j=1}^{i} a_{j}\right)^{\alpha}-\left(\sum_{j=1}^{i} a_{j}\right)^{\alpha}\right] \leq \lambda(\alpha) \cdot\left(\sum_{i=1}^{n} b_{i}\right)^{\alpha}+\mu(\alpha) \cdot\left(\sum_{i=1}^{n} a_{i}\right)^{\alpha}
$$

where $\mu(\alpha)=\frac{\alpha-1}{\alpha}$ and $\lambda(\alpha)=\Theta\left(\alpha^{\alpha-1}\right)$. That implies the competitive ratio $O\left(\alpha^{\alpha}\right)$.

\section{CONCLUSIONS}

This paper considered designing online non-preemptive schedulers - a domain which has long resisted algorithms with strong worst case guarantees. The paper gave provably competitive algorithms in the rejection model. This shows how relaxed models can give rise to good algorithms for the non-preemptive setting. It is of significant interest to develop other realistic relaxations of worst case models (like rejection or resource augmentation) that give rise to strong algorithms for non-preemptive settings.

\section{ACKNOWLEDGMENTS}

Nguyen Kim Thang is supported by the ANR project OATA $n^{0}$ ANR-15-CE40-0015-01, Hadamard PGMO and DIM RFSI. Abhinav Srivastav was supported by the PSL project Multi-Fac and is supported by the ANR project OATA $n^{\circ}$ ANR-15-CE40-0015-01. Benjamin Moseley was supported in part by a Google Research Award, and NSF Grants CCF-1617724, CCF-1733873 and CCF-1725661.

\section{REFERENCES}

[1] Susanne Albers, Evripidis Bampis, Dimitrios Letsios, Giorgio Lucarelli, and Richard Stotz. 2016. Scheduling on power-heterogeneous processors. In Latin American Symposium on Theoretical Informatics. 41-54.

[2] S. Anand, Naveen Garg, and Amit Kumar. 2012. Resource augmentation for weighted flow-time explained by dual fitting. In Symposium on Discrete Algorithms. 1228-1241.

[3] Nikhil Bansal. 2017. Some Open Problems in Scheduling. In Proc. 13th Workshop on Models and Algorithms for Planning and Scheduling Problems.

[4] Nikhil Bansal, Ho-Leung Chan, Dmitriy Katz, and Kirk Pruhs. 2012. Improved Bounds for Speed Scaling in Devices Obeying the Cube-Root Rule. Theory of Computing 8, 1 (2012), 209-229.

[5] Nikhil Bansal, Ho-Leung Chan, and Kirk Pruhs. 2009. Speed scaling with an arbitrary power function. In Proc. 20th ACM-SIAM Symposium on Discrete Algorithms. 693-701.

[6] Nikhil Bansal, Tracy Kimbrel, and Kirk Pruhs. 2007. Speed scaling to manage energy and temperature. F. ACM 54, 1 (2007).

[7] Chandra Chekuri, Sanjeev Khanna, and An Zhu. 2001. Algorithms for minimizing weighted flow time. In Proceedings of the thirty-third annual ACM symposium on Theory of computing. 84-93.

[8] Anamitra Roy Choudhury, Syamantak Das, Naveen Garg, and Amit Kumar. 2015. Rejecting jobs to Minimize Load and Maximum Flow-time. In Proc. Symposium on Discrete Algorithms. 1114-1133.

[9] Johanne Cohen, Christoph Dürr, and Nguyen Kim Thang. 2012. Smooth inequalities and equilibrium inefficiency in scheduling games. In International Workshop on Internet and Network Economics. 350-363.

[10] Nikhil R. Devanur and Zhiyi Huang. 2014. Primal Dual Gives Almost Optimal Energy Efficient Online Algorithms. In Proc. 25th ACM-SIAM Symposium on Discrete Algorithms.

[11] Leah Epstein and Rob van Stee. 2006. Optimal on-line flow time with resource augmentation. Discrete Applied Mathematics 154, 4 (2006), 611-621.

[12] Bala Kalyanasundaram and Kirk Pruhs. 2000. Speed is as powerful as clairvoyance. f. ACM 47, 4 (2000), 617-643.

[13] Hans Kellerer, Thomas Tautenhahn, and Gerhard J. Woeginger. 1999. Approximability and Nonapproximability Results for Minimizing Total Flow Time on a Single Machine. SIAM 7. Comput. 28, 4 (1999), 1155-1166.

[14] Giorgio Lucarelli, Nguyen Kim Thang, Abhinav Srivastav, and Denis Trystram. 2016. Online Non-preemptive Scheduling in a Resource Augmentation Model based on Duality. In European Symposium on Algorithms (ESA), Vol. 57. 1-17.

[15] Cynthia A Phillips, Clifford Stein, Eric Torng, and Joel Wein. 2002. Optimal time-critical scheduling via resource augmentation. Algorithmica 32, 2 (2002), $163-200$.

[16] Nguyen Kim Thang. 2013. Lagrangian Duality in Online Scheduling with Resource Augmentation and Speed Scaling. In Proc. 21st European Symposium on Algorithms. 755-766.

[17] Nguyen Kim Thang. 2017. Online Primal-Dual Algorithms with Configuration Linear Programs. arXiv:1708.04903 (2017).

[18] Frances Yao, Alan Demers, and Scott Shenker. 1995. A scheduling model for reduced CPU energy. In Proceedings. 36th Annual Symposium on Foundations of Computer Science. 374-382. 